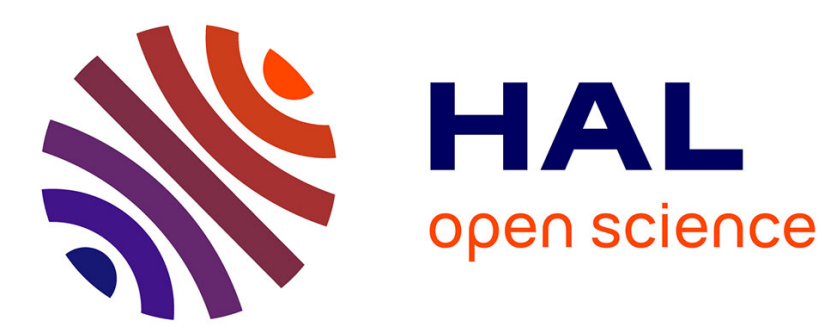

\title{
On the stability and dissipation of wall boundary conditions for compressible flows
}

Nicolas Lamarque, Mauro Porta, Franck Nicoud, Thierry Poinsot

\section{To cite this version:}

Nicolas Lamarque, Mauro Porta, Franck Nicoud, Thierry Poinsot. On the stability and dissipation of wall boundary conditions for compressible flows. International Journal for Numerical Methods in Fluids, 2010, 62 (10), pp.1134-1154. 10.1002/fld.2060 . hal-00802480

\section{HAL Id: hal-00802480 \\ https://hal.science/hal-00802480}

Submitted on 19 Mar 2013

HAL is a multi-disciplinary open access archive for the deposit and dissemination of scientific research documents, whether they are published or not. The documents may come from teaching and research institutions in France or abroad, or from public or private research centers.
L'archive ouverte pluridisciplinaire HAL, est destinée au dépôt et à la diffusion de documents scientifiques de niveau recherche, publiés ou non, émanant des établissements d'enseignement et de recherche français ou étrangers, des laboratoires publics ou privés. 


\title{
On the Stability and Dissipation of Wall Boundary Conditions for Compressible Flows
}

\author{
N. Lamarque ${ }^{1 *}$, M. Porta ${ }^{1}$, F. Nicoud ${ }^{2}$ AND T. Poinsot ${ }^{3}$ \\ ${ }^{1}$ CERFACS, 31057 Toulouse, France \\ ${ }^{2}$ Institut de Mathématiques et de Modélisation de Montpellier, UMR 5149 \\ ${ }^{3}$ Institut de Mécanique des Fluides de Toulouse, UMR 5502
}

\begin{abstract}
SUMMARY
Characteristic formulations for boundary conditions have demonstrated their effectiveness to handle inlets and outlets, especially to avoid acoustic wave reflections. At walls, however, most authors use simple Dirichlet or Neumann boundary conditions, where the normal velocity (or pressure gradient) is set to zero. This paper demonstrates that there are significant differences between characteristic and Dirichlet methods at a wall and that simulations are more stable when using walls modelled with a characteristic wave decomposition. The derivation of characteristic methods yields an additional boundary term in the continuity equation, which explains their increased stability. This term also allows to handle the two acoustic waves going towards and away from the wall in a consistent manner. Those observations are first confirmed by one-dimensional simulations and stability matrix analysis of acoustic modes in cavities. Finally, a two-dimensional test case shows the validity of the demonstration in multi-dimensional configurations. Copyright (C) 2000 John Wiley \& Sons, Ltd.
\end{abstract}

\footnotetext{
*Correspondence to: nicolas.lamarque2@cea.fr - now at SSTH/LMDL, CEA-Grenoble, 38041 GRENOBLE CEDEX
} 
KEY WORDS: Dirichlet and characteristic boundary conditions, additional boundary condition term, increased stability, matrix analysis.

\section{INTRODUCTION}

Many aspects of turbulent compressible flows can be simulated with the help of Large Eddy Simulation (LES), which solves the filtered unsteady Navier-Stokes (NS) equations [1, 2, 3, 4]. The increasing available computational power allows to carry out LES in more and more complex flows and geometries $[6,7,8,9,10]$. One of the key issues to ensure the quality of the resolution is the accuracy of the numerical scheme used to discretize the convective terms $[11,12,13,14]$. Families of discretizations - such as compact schemes $[15,16,17]$ or finite differences with summation-by-parts (SBP) properties [18] on structured grids, or TaylorGalerkin (TG) schemes [12, 19] on unstructured meshes - have demonstrated their abilities to give satisfactory results. The treatment of the boundary conditions (BC) has also been a subject of intense research $[20,21,22,23,24,25]$ since it can dramatically deteriorate both the accuracy and robustness of the calculations. Non-reflecting BC based on characteristic treatment (wave analysis at boundaries) have been developed and are now commonly used to model inlets and outlets of the computational domain and their influence on acoustics is now well identified [26, 27, 28, 29]. Despite the proven effectiveness of characteristic formulations, walls are still often represented with Dirichlet BC, where the velocity is set to zero and density at the wall is obtained either using the continuity equation without characteristic treatment or specifying a zero pressure gradient. The behaviour of wall $\mathrm{BC}$ is a critical issue to study acoustic damping in cavities (such as combustion chambers) and controlling the dissipation 
and dispersion of acoustic waves at walls is required in many present research fields such as combustion instability studies [14, 30, 31]. This paper presents an analysis of wall BC using both Dirichlet and characteristic methods. Despite the apparent simplicity of specifying a wall BC, results show that characteristic methods differ from Dirichlet techniques at walls and provide more stable schemes. This is confirmed by a linear stability analysis incorporating wall BC. Throughout the article, wall means any rigid obstacle in the computational domain (on the sides or inside the domain).

The formalism used for characteristic BC is first recalled (section 2) and compared to Dirichlet BC in the case of a solid wall (section 3). Characteristic formulations lead to the introduction of an additional $\mathrm{BC}$ term (called the $\mathrm{ABC}$ term), supposed to be zero in the continuous problem but not in its discretized counterpart. This term increases the stability of the scheme, while not degrading its accuracy. A simple test case (one-dimensional acoustic cavity) is then used to study the influence of the additional term (section 4). Simulations and matrix analyses confirm the theoretical observations, showing the importance of considering characteristic treatment for the hyperbolic part of wall BC (section 5). Finally, both wall $\mathrm{BC}$ are used to simulate a longitudinal / transversal 2D acoustic mode in an infinite duct to demonstrate the conclusions are also valid in multidimensional applications (section 6).

\section{CHARACTERISTIC BOUNDARY CONDITIONS}

The present description uses the Navier Stokes Characteristic Boundary Conditions (NSCBC) method $[21,32,33]$ as a prototype for characteristic methods but the results below hold for most characteristic BC. The NSCBC method is a standard technique for compressible flows, which enables to correctly handle the hyperbolic part of the NS equations [20, 21, 24, 34]. The 
NS equations are first recast, in a new direct orthonormal basis $\left(\vec{n}, \vec{t}_{1}, \vec{t}_{2}\right)$, in the quasi-linear form with primitive variables: $\mathbf{V}=\left(\rho, u_{x}, u_{y}, u_{z}, p\right)^{T}$ with $\rho$ the density, $\left(u_{x}, u_{y}, u_{z}\right)$ the $x$, $y$ and $z$ velocity components and $p$ the pressure. The $\vec{n}$ corresponds to the outward normal vector on boundaries $\partial \Omega$. The new set of equations is written:

$$
\frac{\partial \mathbf{V}}{\partial t}+\left(\overrightarrow{\mathcal{A}}_{\mathbf{V}} \cdot \vec{n}\right) \frac{\partial \mathbf{V}}{\partial n}=\mathbf{T}
$$

where $\overrightarrow{\mathcal{A}}_{\mathbf{V}}$ is the Jacobian tensor of the primitive variables and $\mathbf{T}$ represents all other contributions (tangential, diffusion and source terms). Matrix $\overrightarrow{\mathcal{A}}_{\mathbf{V}} \cdot \vec{n}$ is diagonalizable and (1) is strictly hyperbolic. If $\mathbf{W}$ are the characteristic variables, then :

$$
\frac{\partial \mathbf{W}}{\partial t}+\Lambda \frac{\partial \mathbf{W}}{\partial n}=\mathbf{T}_{\mathbf{W}}
$$

and:

$$
\Lambda=\operatorname{diag}\left(\lambda_{+}, \lambda_{-}, \lambda_{t_{1}}, \lambda_{t_{2}}, \lambda_{S}\right)=\operatorname{diag}\left(u_{n}+c, u_{n}-c, u_{n}, u_{n}, u_{n}\right)
$$

where $\Lambda$ is the diagonal matrix containing the velocities of the characteristic waves $\partial \mathbf{W}$, while

$\mathbf{T}_{\mathbf{W}}$ contains the other contributions. We will use in the following the notation $\mathcal{L}$ of $[21]$ for the wave amplitude variations:

$$
\Lambda \frac{\partial \mathbf{W}}{\partial n}=\left(\begin{array}{c}
\mathcal{L}_{+} \\
\mathcal{L}_{-} \\
\mathcal{L}_{t_{1}} \\
\mathcal{L}_{t_{2}} \\
\mathcal{L}_{S}
\end{array}\right)=\left(\begin{array}{c}
\left(u_{n}+c\right)\left(\frac{\partial u_{n}}{\partial n}+\frac{1}{\rho c} \frac{\partial p}{\partial n}\right) \\
\left(u_{n}-c\right)\left(\frac{\partial u_{n}}{\partial n}-\frac{1}{\rho c} \frac{\partial p}{\partial n}\right) \\
u_{n} \frac{\partial u_{t_{1}}}{\partial n} \\
u_{n} \frac{\partial u_{t_{2}}}{\partial n} \\
u_{n}\left(-\frac{1}{c^{2}} \frac{\partial p}{\partial n}+\frac{\partial \rho}{\partial n}\right)
\end{array}\right)
$$


where $u_{n}, u_{t_{1}}$ and $u_{t_{2}}$ are the components of the velocity in $\left(\vec{n}, \overrightarrow{t_{1}}, \overrightarrow{t_{2}}\right)$ and $c$ is the local speed of sound.

At the boundaries $\partial \Omega$, ingoing and outgoing waves are identified. The outgoing waves come from the inner domain $\Omega$. Thus, they are not to be modified. On the other hand, ingoing waves supply information from the outside and enable the imposition of $\mathrm{BC}$ for the problem to be solved. The NSCBC method is based on the use of the LODI (for Local One-Dimensional Inviscid) relations [21]. LODI relations allow to formulate the incoming wave amplitudes, depending on the BC. One should note that characteristic methods are better suited for linear small perturbations (such as acoustic or entropy waves) and have sometimes difficulties with non-linear phenomena [35]. At a wall (Figure 1), the entropy wave and the shear waves are zero: $\mathcal{L}_{S}=\mathcal{L}_{t_{1}}=\mathcal{L}_{t_{2}}$. The LODI relation for velocity shows that the ingoing wave $\mathcal{L}_{+}$must be such that: $\mathcal{L}_{+}=+\mathcal{L}_{-}$. Note that the wave $\mathcal{L}_{+}$enters the domain and should not be evaluated with data obtained within the domain.

A complete set of BC is described in [21, 25, 32, 36, 29] and generalization to multi-component flows is given, for instance, in [33, 37]. The clear superiority of characteristic methods to model partially or non-reflecting inlets or outlets is a theme vastly treated in the literature [21, 34]. Here, we focus on the special case of solid walls (Figure 1), for which many authors use simpler methods such as Dirichlet BC.

Of course, both Dirichlet and characteristic methods eventually impose a zero velocity at the wall. The differences between methods come from the evaluation of pressure, density and temperature at the wall. Table I summarizes the two methods for an adiabatic wall.

Copyright (c) 2000 John Wiley \& Sons, Ltd.

Int. J. Numer. Meth. Fluids 2000; 00:1-6

Prepared using fldauth.cls 


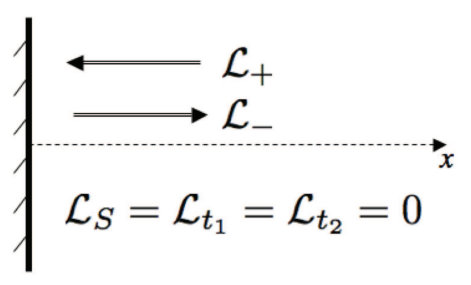

Figure 1. Characteristic waves at a wall.

\begin{tabular}{|c||c|c|}
\hline & Dirichlet & NSCBC \\
\hline Velocity & $u_{n}=0$ & $\mathcal{L}^{-}=\mathcal{L}^{+} \Rightarrow \partial_{t} u_{n}=0 \Rightarrow u_{n}=0$ \\
\hline Density & obtained from full continuity & obtained from full continuity equation \\
& equation with $u_{n}=0$ & AFTER ingoing wave modification \\
\hline Pressure & obtained from full continuity and & obtained from pressure equation \\
& energy equations with $u_{n}=0$ & AFTER ingoing wave modification \\
\hline Temperature & deduced from state equation & obtained from temperature equation \\
& & AFTER ingoing wave modification \\
\hline
\end{tabular}

Table I. Differences between Dirichlet and NSCBC methods

\section{WALL TREATMENT}

To illustrate the differences between Dirichlet and characteristic BC, it is sufficient to consider isentropic cases. Therefore the following analysis focuses on isentropic flows surrounded by adiabatic walls. The conclusions are the same for non-isentropic flows.

\subsection{Linearized Euler equations}

The differences between Dirichlet and characteristic formulations for the walls are now brought into focus. In the following, we consider one-dimensional flow to simplify the analysis. Therefore, the existing waves are the acoustic waves $\mathcal{L}_{+}$and $\mathcal{L}_{-}$(Figure 1 ). To understand Copyright (C) 2000 John Wiley \& Sons, Ltd. Int. J. Numer. Meth. Fluids 2000; 00:1-6 Prepared using fldauth.cls 
how a full NS solver using Dirichlet or characteristic BC will perform, it is useful to linearize the Euler equations around the state: $(\rho, u)^{T}=(\bar{\rho}, 0)^{T}$ :

$$
\begin{aligned}
\frac{\partial \rho^{\prime}}{\partial t}+\frac{\partial}{\partial x}\left(\bar{\rho} u^{\prime}\right) & =0 \\
\frac{\partial \bar{\rho} u^{\prime}}{\partial t}+\frac{\partial p^{\prime}}{\partial x} & =0 .
\end{aligned}
$$

The primed values refer to fluctuations around the mean state noted with an overbar. As the flow is isentropic: $p^{\prime}=\rho^{\prime} c^{2}$ and the system of Eqs (5)-(6) can be recast:

$$
\frac{\partial}{\partial t}\left(\begin{array}{c}
\rho^{\prime} \\
u^{\prime}
\end{array}\right)+\left(\begin{array}{cc}
0 & \bar{\rho} \\
\frac{c^{2}}{\bar{\rho}} & 0
\end{array}\right) \frac{\partial}{\partial x}\left(\begin{array}{c}
\rho^{\prime} \\
u^{\prime}
\end{array}\right)=\left(\begin{array}{l}
0 \\
0
\end{array}\right)
$$

or in matrix form:

$$
\frac{\partial \mathbf{U}}{\partial t}+A \frac{\partial \mathbf{U}}{\partial x}=\mathbf{0}
$$

where $A$ is the flux Jacobian matrix.

\subsection{Walls with Dirichlet formulation}

Solid walls are usually treated with Dirichlet BC:

$$
\vec{u} \cdot \vec{n}=u_{n}=0,
$$

with $\vec{u}$ the velocity vector and $\vec{n}$ the outward pointing normal. At the wall, the continuity equation becomes:

$$
\frac{\partial \rho}{\partial t}=-(\underbrace{u_{n} \frac{\partial \rho}{\partial n}}_{=0}+\rho \frac{\partial u_{n}}{\partial n})=-\rho \frac{\partial u_{n}}{\partial n} .
$$

Eq (10) is then used to obtain the density value at the walls. This is the value predicted by the scheme at the wall. 
It should be noted here that the same set of equations would have been obtained by imposing a zero normal pressure gradient $\frac{\partial p}{\partial n}=0$ (Neumann condition), instead of a zero normal velocity. Indeed, using this $\mathrm{BC}$, the initial condition at the wall $\left(u_{n}(t=0)=0\right)$ and $\mathrm{Eq}(6)$ yields:

$$
\frac{\partial p}{\partial n}=0 \quad \text { and } \quad u_{n}(t=0)=0 \quad \Longrightarrow \quad u_{n}=0,
$$

which is Eq (9). Then, as $u_{n}=0$ and $\partial p / \partial n=0$, Eq (5) becomes Eq (10). Therefore, Dirichlet $\mathrm{BC}$ or zero pressure gradient $\mathrm{BC}$ are equivalent on an adiabatic wall.

\subsection{Walls with characteristic formulation}

The characteristic formulation of NSCBC (Figure 1) makes use of the following LODI relations for normal velocity and density:

$$
\begin{aligned}
\frac{\partial u_{n}}{\partial t}+\frac{1}{2}\left(\mathcal{L}_{+}-\mathcal{L}_{-}\right) & =0, \\
\frac{\partial \rho}{\partial t}+\frac{\rho}{2 c}\left(\mathcal{L}_{+}+\mathcal{L}_{-}\right)+\mathcal{L}_{S} & =0
\end{aligned}
$$

From an acoustic point of view, a solid wall is a totally reflecting solid surface (zero admittance) that imposes: $\mathcal{L}_{+}=\mathcal{L}_{-}$and $\mathcal{L}_{S}=0$ (isentropic flow).

It is interesting to compare Eqs (9)-(10) with Eqs (12)-(13). Equation (12) with $\mathcal{L}_{+}=\mathcal{L}_{-}$ entails:

$$
u_{n}(t)=u_{n}(t=0)=0
$$

which is equivalent to equation (9), as long as the initial condition is $u_{n}(t=0)=0$ at the wall.

On the other hand, using relations (4), equation (13) leads to : 


$$
\frac{\partial \rho}{\partial t}+\left(\rho \frac{\partial u_{n}}{\partial n}+\frac{1}{c} \frac{\partial p}{\partial n}\right)=0 .
$$

Physically, a zero admittance implies $\partial p / \partial n=0$ on a wall. Therefore, Eqs (10) and (15) should be physically equivalent at the wall. From a discrete point of view however, derivatives $(\partial / \partial n)$ are replaced by differences $(\delta / \delta n)$. A simple consequence is that, most often:

$$
\left.\frac{\delta p}{\delta n}\right|_{\partial \Omega} \neq 0
$$

where $\delta / \delta n$ is the discrete first-order derivative operator. Thus, the discrete counterparts of Eqs (10) and (13) are not equivalent (Table II). We will see later that the added term $\delta p /\left.\delta n\right|_{\partial \Omega}$ (called here Additional Boundary Condition (ABC) term) has an important stabilization role.

\begin{tabular}{|l||c|c|}
\hline & Dirichlet & NSCBC \\
\hline Velocity & $u_{n}=0$ & $\mathcal{L}^{-}=\mathcal{L}^{+} \Rightarrow \partial_{t} u_{n}=0 \Rightarrow u_{n}=0$ \\
\hline Density & obtained from $\frac{\partial \rho}{\partial t}=-\rho \frac{\partial u}{\partial n}$ & obtained from $\frac{\partial \rho}{\partial t}=-\rho \frac{\partial u}{\partial n}-\underbrace{c \frac{\partial \rho}{\partial n}}_{\text {ABC term }}$ \\
\hline
\end{tabular}

Table II. Equations used to advance wall values in Dirichlet and NSCBC methods

\section{A SIMPLE TEST FOR WALL BOUNDARY CONDITIONS}

This section presents a simple one-dimensional numerical setup which allows to verify the stability and dissipation of BC formulations at walls. 


\subsection{Numerical setup}

Consider an acoustic eigenmode between two walls in a one-dimensional cavity. Figure 2 gives a simple sketch of the problem. The mean state is such that: $(\bar{\rho}, 0)^{T}=$ cst and $A=$ cst in $\mathrm{Eq}(8)$.

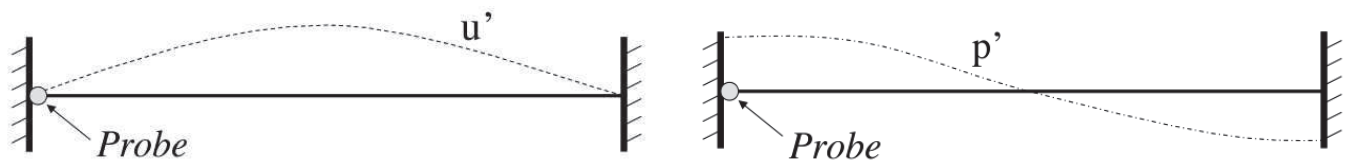

Figure 2. Sketch of the one dimensional cavity problem. Left: instantaneous velocity at $t=k T$. Right: instantaneous pressure at $t=3 T / 2+k T$, with $k \in \mathbb{N}$.

The initial conditions of the problem are:

$$
\left\{\begin{array}{l}
p^{\prime}(x, t=0)=\rho^{\prime}(x, t=0)=0, \\
u^{\prime}(x, t=0)=\frac{p_{0}}{\bar{\rho} c} \sin \left(\frac{\pi x}{L}\right),
\end{array}\right.
$$

with $L=1 \mathrm{~m}$ and $p_{0}=\frac{\bar{p}}{100}$. All values are non-dimensionalized and the mode period is $T=2 L / c$. The exact solution for pressure in absence of viscous dissipation is then:

$$
p^{\prime}(x, t)=-p_{0} \cos \left(\frac{\pi x}{L}\right) \sin \left(\frac{\pi c t}{L}\right)
$$

\subsection{Taylor-Galerkin discretization}

The chosen numerical scheme in this study belongs to the Taylor-Galerkin family of discretizations, as they are often chosen to carry out LES in complex geometries with unstructured meshes [3, 14]. Those schemes have been developed adapting the ideas of Lax and Wendroff in the context of finite elements [19]. The classical Galerkin centred-in-space scheme has very good dispersion properties but is also unconditionally unstable, when associated Copyright @ 2000 John Wiley \& Sons, Ltd. Int. J. Numer. Meth. Fluids 2000; 00:1-6 Prepared using fldauth.cls 
explicit Euler time marching method [19]. Therefore, stabilization is needed and obtained by reaching higher-order time accuracy. To do this, the solution is first expanded with Taylor series in time and time derivatives are replaced by space ones. Then, Galerkin space discretization is used [19]. The resulting full discretizations are often cheaper, more accurate and more stable than classical centred schemes with explicit Runge-Kutta or semi-implicit Crank-Nicolson time marching methods in the context of turbulent flows in complex geometries.

4.2.1. Inner Domain The domain $\Omega=[0,1]$ is spatially discretized in $N$ regular cells and $h$ is the step in space. System (7) is discretized using the second-order TG scheme [19, 38, 39] with P1 linear elements, which is a classical numerical scheme used with unstructured grids, secondorder in space and time. The mass matrix is lumped to simplify the study. The application of the lumped TG scheme (noted TG2 throughout the paper) to equation (7) yields:

$$
\left(\mathbf{U}_{I}^{n+1}-\mathbf{U}_{I}^{n+1}\right) h=-\left.\Delta t \int_{\Omega} A \frac{d \mathbf{U}_{h}}{d x}\right|^{n} N_{I} d x+\frac{\Delta t^{2}}{2} \int_{\Omega} A \frac{d}{d x}\left(\left.A \frac{d \mathbf{U}_{h}}{d x}\right|^{n}\right) N_{I} d x
$$

where:

$$
\mathbf{U}_{h}^{n}(x)=\sum_{J} \mathbf{U}_{J}^{n} N_{J}(x)
$$

and $N_{I}$ is the hat shape function associated with node $I$. Eq (19) gives with classical finite differences operators:

$$
\begin{aligned}
& \rho_{I}^{n+1}=\rho_{I}^{n}-\frac{\bar{\rho} \Delta t}{h} \Delta_{0} u_{I}^{n}+\frac{1}{2} \frac{c^{2} \Delta t^{2}}{h^{2}} \delta^{2} \rho_{I}^{n} \\
& u_{I}^{n+1}=u_{I}^{n}-\frac{c^{2}}{\bar{\rho}} \frac{\Delta t}{h} \Delta_{0} \rho_{I}^{n}+\frac{1}{2} \frac{c^{2} \Delta t^{2}}{h^{2}} \delta^{2} u_{I}^{n}
\end{aligned}
$$

where:

Copyright (C) 2000 John Wiley \& Sons, Ltd.

Int. J. Numer. Meth. Fluids 2000; 00:1-6

Prepared using fldauth.cls 


$$
\Delta_{0} u_{I}^{n}=\frac{1}{2}\left(u_{I+1}^{n}-u_{I-1}^{n}\right) \quad \text { and } \quad \delta^{2} u_{I}^{n}=u_{I+1}^{n}-2 u_{I}^{n}+u_{I-1}^{n}
$$

As the computational grid is regular here, a more classical Lax-Wendroff finite difference scheme [40] would have also given Eqs (20) and (21) for the inner domain.

4.2.2. Numerical boundary schemes - First derivative On the boundaries, differences are not centred anymore. The simplest and most usual approximations of the first derivatives are therefore ${ }^{\dagger}$ :

$$
\begin{gathered}
\left.\frac{\partial \mathbf{U}}{\partial x}\right|_{0} \approx \frac{1}{h} \delta^{+} \mathbf{U}_{0}=\frac{1}{h}\left(\mathbf{U}_{1}-\mathbf{U}_{0}\right) \quad \text { and }\left.\quad \frac{\partial \mathbf{U}}{\partial n}\right|_{0}=-\left.\frac{\partial \mathbf{U}}{\partial x}\right|_{0} \\
\left.\frac{\partial \mathbf{U}}{\partial x}\right|_{N} \approx \frac{1}{h} \delta^{-} \mathbf{U}_{N}=\frac{1}{h}\left(\mathbf{U}_{N}-\mathbf{U}_{N-1}\right) \quad \text { and }\left.\quad \frac{\partial \mathbf{U}}{\partial n}\right|_{N}=+\left.\frac{\partial \mathbf{U}}{\partial x}\right|_{N}
\end{gathered}
$$

and $\mathbf{U}_{0}$ and $\mathbf{U}_{N}$ can be imposed as boundary conditions.

4.2.3. Numerical boundary schemes - Second derivative The case of the second derivative is more complicated. Integration by parts of the last term of Eq (19) yields at node $I$ :

$$
L L_{I}\left(\mathbf{U}^{n}\right)=\int_{\Omega} \frac{d}{d x}\left(\left.c^{2} \frac{d \mathbf{U}_{h}}{d x}\right|^{n}\right) N_{I} d x=\underbrace{\left[\left.c^{2} \frac{d \mathbf{U}_{h}}{d x}\right|^{n} N_{I}\right]_{x=0}^{x=L}}_{B T_{I}\left(\mathbf{U}^{n}\right)} \underbrace{-\left.\int_{\Omega} c^{2} \frac{d \mathbf{U}_{h}}{d x}\right|^{n} \frac{d N_{I}}{d x} d x}_{L L_{I}^{i n}\left(\mathbf{U}^{n}\right)}
$$

If $I \in \Omega \backslash \partial \Omega$, then $B T_{I}\left(\mathbf{U}^{n}\right)=0$ and $L L_{I}\left(\mathbf{U}^{n}\right)=\frac{c^{2}}{h} \delta^{2} \mathbf{U}_{I}^{n}$, which corresponds to the second terms of the right hand sides of Eq (20) and (21). On the other hand, if $I \in \partial \Omega$, which gives

\footnotetext{
${ }^{\dagger}$ Higher-order evaluations of derivatives can be constructed using larger stencils but this is difficult and expensive on unstructured meshes.

Copyright (c) 2000 John Wiley \& Sons, Ltd.

Int. J. Numer. Meth. Fluids 2000; 00:1-6

Prepared using fldauth.cls
} 
$I=0$ or $I=N$, then $B T_{I}\left(\mathbf{U}^{n}\right)$ has to be calculated. The case of inviscid flows arises a difficulty, as there is no natural boundary conditions for $\left.\frac{d \mathbf{U}_{h}}{d x}\right|_{\partial \Omega}$ to express $B T_{I}\left(\mathbf{U}^{n}\right)$, as for parabolic or elliptic problems. As we deal with linear elements, first derivatives are constant inside an element. The choice is then to consider that $\left.\frac{d \mathbf{U}_{h}}{d x}\right|_{\partial \Omega}$ is equal to $\frac{d \mathbf{U}_{h}}{d x}$ inside the element adjacent to the boundary. Therefore, using Eq (25), this is equivalent to set $L L_{0}$ and $L L_{N}$ to zero, which is equivalent to use first-order explicit Euler time marching instead of Lax-Wendroff method for the temporal integration at the boundaries. For instance, at node 0, using Eq (25), the numerical boundary scheme is:

$$
\begin{aligned}
L L_{0}\left(\mathbf{U}^{n}\right) & =B T_{0}\left(\mathbf{U}^{n}\right)+L L_{0}^{i n}\left(\mathbf{U}^{n}\right) \\
& =-\frac{c^{2}}{2 h}\left(\mathbf{U}_{1}^{n}-\mathbf{U}_{0}^{n}\right)-\frac{c^{2}}{2 h}\left(\mathbf{U}_{1}^{n}-\mathbf{U}_{0}^{n}\right) \frac{-1}{h} h \\
& =0
\end{aligned}
$$

4.2.4. Boundary conditions For Dirichlet boundary condition, the discretization at the boundaries is then:

$$
\begin{aligned}
\rho_{0}^{n+1} & =\rho_{0}^{n}-\frac{\bar{\rho} \Delta t}{h}\left(u_{1}^{n}-u_{0}^{n}\right) \\
\rho_{N}^{n+1} & =\rho_{N}^{n}-\frac{\bar{\rho} \Delta t}{h}\left(u_{N}^{n}-u_{N-1}^{n}\right)
\end{aligned}
$$

$u_{0}$ and $u_{N}$ are imposed to be zero.

For characteristic BC, the discretizations of Eqs (14)-(15) are:

Copyright (c) 2000 John Wiley \& Sons, Ltd.

Int. J. Numer. Meth. Fluids 2000; 00:1-6

Prepared using fldauth.cls 


$$
\begin{aligned}
\rho_{0}^{n+1} & =\rho_{0}^{n}-\frac{\bar{\rho} \Delta t}{h}\left(u_{1}^{n}-u_{0}^{n}\right)+\frac{c \Delta t}{h}\left(\rho_{1}^{n}-\rho_{0}^{n}\right) \\
u_{0}^{n+1} & =u_{0}^{n} \\
\rho_{N}^{n+1} & =\rho_{N}^{n}-\frac{\bar{\rho} \Delta t}{h}\left(u_{N}^{n}-u_{N-1}^{n}\right)-\frac{c \Delta t}{h}\left(\rho_{N}^{n}-\rho_{N-1}^{n}\right) \\
u_{N}^{n+1} & =u_{N}^{n}
\end{aligned}
$$

As $u_{0}^{0}=0$ and $u_{N}^{0}=0$, the only differences between Eqs (29)-(30) and Eqs (31)-(34) are the $\mathrm{ABC}$ terms in the continuity equation for characteristic BC.

\section{RESULTS}

In this section, the influence of the ABC terms is studied using two methods: (1) comparison of amplification matrices and (2) comparison of simulation results. For both methods, the test case is the $1 \mathrm{D}$ acoustic eigenmode of Figure 2.

\subsection{Amplification matrices}

Let $\mathbf{U}$ be the solution vector containing the degrees of freedom of the problem (the velocity and pressure at all grid points). The amplification matrix $Q$ is defined by [41]:

$$
\mathbf{U}^{n+1}=Q \mathbf{U}^{n}
$$

and $^{\ddagger} \mathbf{U}^{n}=\left(\rho_{0}^{n}, u_{0}^{n}, \ldots, \rho_{N}^{n}, u_{N}^{n}\right)^{T}$. For the present problem, appendix A gives all terms of the matrix for both methods (Dirichlet and characteristic). To be stable, it is necessary for the

\footnotetext{
${ }_{\ddagger}^{\ddagger}$ It should be noted that $u_{0}^{n}$ and $u_{N}^{n}$ do not belong to $Q$ in the case of Dirichlet BC, as they are fixed. Copyright (c) 2000 John Wiley \& Sons, Ltd. Int. J. Numer. Meth. Fluids 2000; 00:1-6 Prepared using fldauth.cls
} 
spectral radius $\rho(Q)$ to be less than or equal ${ }^{\S}$ to 1 [41]. Indeed the initial solution can be decomposed in a sum of eigenvectors $\mathbf{V}_{i}$ :

$$
\mathbf{U}^{0}=\sum_{i=0}^{N} \alpha_{i} \mathbf{V}_{i}
$$

Then, solution vector at time $t=(n+1) \Delta t$ is:

$$
\mathbf{U}^{n+1}=Q \mathbf{U}^{0}=\sum_{i=0}^{N} \lambda_{i}^{n} \alpha_{i} \mathbf{V}_{i} \quad \propto \quad \lambda_{K}^{n} \alpha_{K} \mathbf{V}_{K}+O . T
$$

where $\lambda_{i}$ is the eigenvalue associated with $\mathbf{V}_{i}$ and $K$ is the index of the eigenvalue, which has the greater modulus (except if $\left|\lambda_{K}\right|=1$ ). O.T contains all the other terms, including those with $\lambda_{i}=1$ (corresponding to the steady state part of solution). Eq (37) simply shows that the long term behaviour of $\mathbf{U}$ is similar to $\lambda_{K}^{n} \alpha_{K} \mathbf{V}_{K}$ and will lead to instability if $\left|\lambda_{K}\right|>1$.

Figures 3 and 4 show the spectra in complex plane, obtained with Dirichlet and characteristic BC. The mesh contains 15 points. Stability domain is represented by the unit circle centered around origin. If an eigenvalue lies outside it, then the method is unstable. Moreover, one should expect that for big enough $n,\left|\lambda_{K}\right|^{n}$ gives the damping / amplification factor of the signal. It also implies that the closer to the unit circle the eigenvalues are, the less dissipative the discretization is.

In the studied cases (with TG2 scheme), the complete discretization is unconditionally unstable when using Dirichlet BC, as there are some eigenvalues with a modulus greater than one, for all CFL values. On the other hand, it is conditionally stable with characteristic BC and the stability limit is given by the CFL number: $\nu=c \Delta t / h \leq 1$. Besides, the eigenvalues also

\footnotetext{
$\S$ The condition for Lax-Richtmeyer stability is: $\rho(Q) \leq 1+\varepsilon / N(\varepsilon$ strictly positive and not depending on $N)$ [42], but in the cases studied here, it is enough to use the condition $\rho(Q) \leq 1$.

Copyright (c) 2000 John Wiley \& Sons, Ltd.

Int. J. Numer. Meth. Fluids 2000; 00:1-6

Prepared using fldauth.cls
} 
globally lie further from the unit circle than those obtained with Dirichlet BC. Consequently, as expected, the ABC terms have a stabilizing effect.
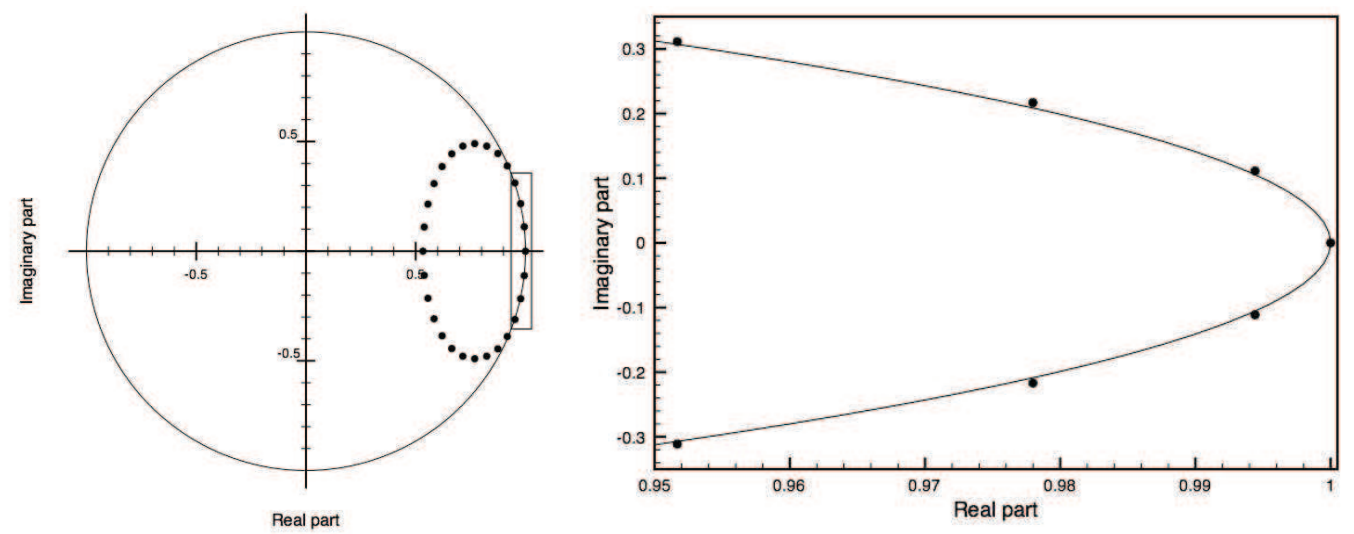

Figure 3. Left: complete spectrum of amplification matrix $Q$ for Dirichlet BC and 15 mesh nodes. Right: zoom on unstable eigenvalues corresponding to the box of the left figure. CFL number: 0.5 .
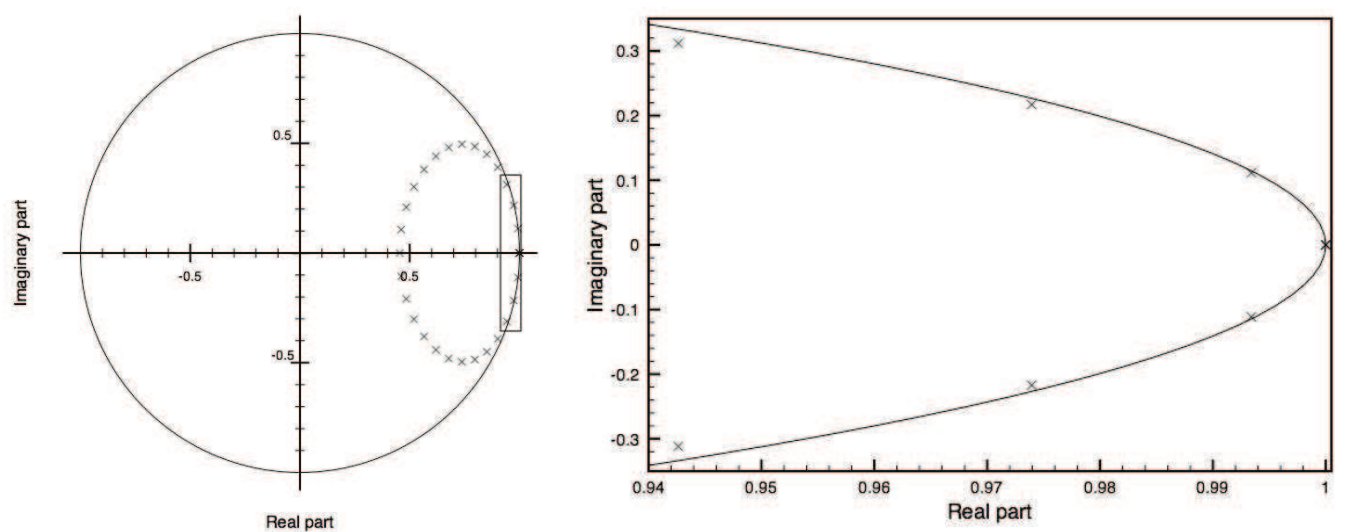

Figure 4. Left: complete spectrum of amplification matrix $Q$ for NSCBC and 15 mesh nodes. Right: zoom on unstable eigenvalues corresponding to the box of the left figure. CFL number: 0.5 . 


\subsection{Simulations}

The test case of figure 2 is now run in a DNS code using TG2 scheme and both BC are compared. Figure 5 first presents the temporal variation of pressure fluctuation for Dirichlet $\mathrm{BC}$, while figure 6 gives the same results for characteristic $\mathrm{BC}$ at the probe indicated on figure 2 on two different meshes ${ }^{\top}$. As predicted by the amplification matrix analysis of section 5.1 , the pressure signal diverges when using Dirichlet BC, whereas it is damped with NSCBC, hence ensuring the stability of the problem. It is also evident that refining the mesh limits the damping effect due to NSCBC. It should also be added that, as suggested by the matrix analysis, the largest eigenvalue controls the envelope of the pressure fluctuation: the signal $\mathbf{U}^{n}$ behaves like $\lambda_{K}^{n} \alpha_{K} \mathbf{V}_{K}$ as shown by Eq (37). This simple numerical simulation case thus confirms the stabilization induced by the added pressure term.

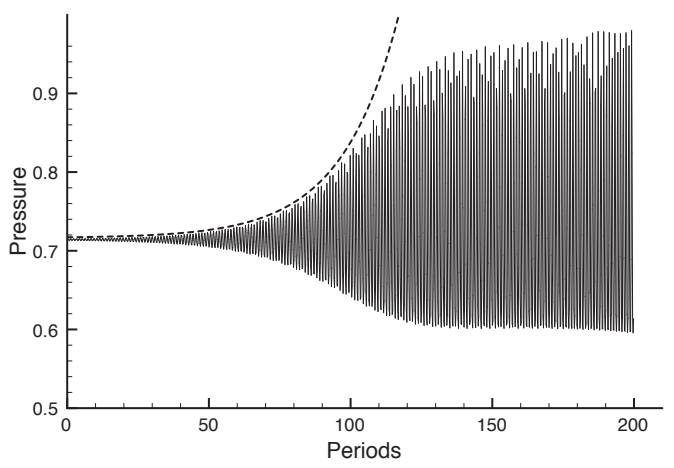

Figure 5. Pressure fluctuations at the probe of Fig. 2. Dirichlet BC with 15 mesh nodes. (Plain line: simulation, dash line: prediction (Eq.(37)) with $\left|\lambda_{K}\right|$ ). CFL number: 0.5 .

\footnotetext{
『As the simulations always diverge with Dirichlet BC, only one case is presented. 

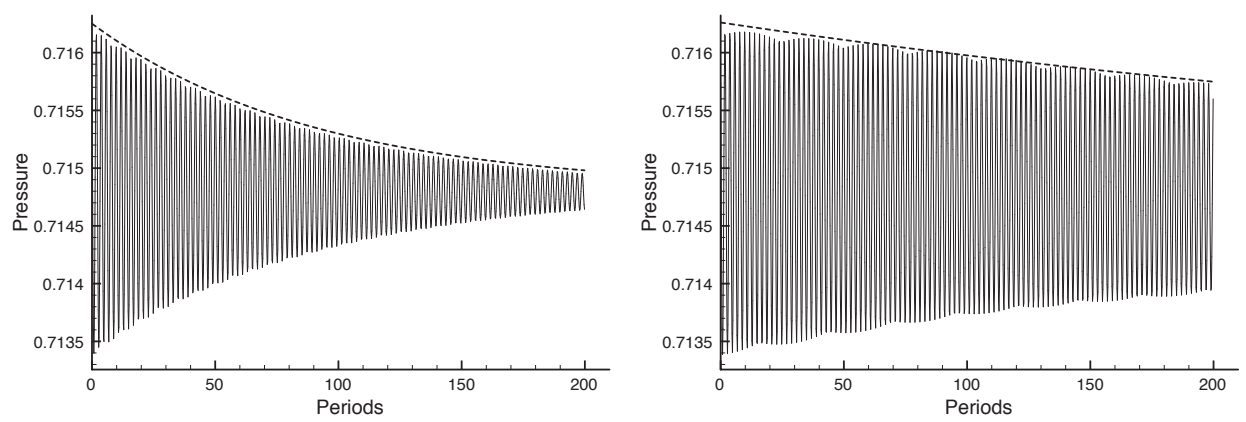

Figure 6. Pressure fluctuations at the probe of Fig. 2. NSCBC with 15 (left) and 31 (right) mesh nodes. (Plain line: simulation, dash line: prediction (Eq.(37)) with $\left|\lambda_{K}\right|$ ). CFL number: 0.5 .

\subsection{Further analysis}

Accuracy of $A B C$ terms It is interesting to focus now on the ABC terms and highlight why they stabilize the calculations. A first reason is that these terms are clearly generated when a characteristic analysis is used in the momentum equation. Using simply the continuity equation and failing to identify waves as done in the Dirichlet method means that the acoustic wave entering the domain through the wall $\left(\mathcal{L}_{+}\right.$in Eq $\left.(4)\right)$ is computed using downwind differencing which is intrinsically unstable. Sections 5.1 and 5.2 essentially recover this expected unstable behaviour. A second reason can be provided by expanding the $\mathrm{ABC}$ terms as follows:

$$
\begin{aligned}
-\left.c \frac{\delta \rho}{\delta n}\right|_{x=0} & =\left.c \frac{\partial \rho}{\partial x}\right|_{x=0}+\left.\frac{1}{2} \operatorname{ch} \frac{\partial^{2} \rho}{\partial x^{2}}\right|_{x=0}+\mathcal{O}\left(h^{2}\right) \\
-\left.c \frac{\delta \rho}{\delta n}\right|_{x=L} & =-\left.c \frac{\partial \rho}{\partial x}\right|_{x=L}+\left.\frac{1}{2} \operatorname{ch} \frac{\partial^{2} \rho}{\partial x^{2}}\right|_{x=L}+\mathcal{O}\left(h^{2}\right)
\end{aligned}
$$

Eqs (38) and (39) shows that using these non-centered differences to express characteristic waves $\mathcal{L}$ implies the addition of a diffusive term $\frac{1}{2} \operatorname{ch} \frac{\partial^{2} \rho}{\partial x^{2}}$ in the continuity equation. This also explains the origin of the stabilization induced by NSCBC.

The inner local order of accuracy is 2 . Theoretically, even though boundary derivatives are Copyright (c) 2000 John Wiley \& Sons, Ltd. Int. J. Numer. Meth. Fluids 2000; 00:1-6 Prepared using fldauth.cls 
first-order accurate, the global convergence rate remains second-order [43]. The question is then whether this additional term deteriorates the global accuracy of the calculation, as it is also first-order accurate. Simulations, using 9, 11, 15, 31, 45 and 61 mesh nodes, have been carried out to measure the global error on density:

$$
L^{2}\left(\epsilon_{\rho}\right)=\sum_{i=0}^{N}\left|\rho_{i}-\rho^{\text {exact }}\left(x_{i}\right)\right|^{2}
$$

Figure 7 indicates that the $L^{2}$-norm of the density error is close to 2, proving that the additional term does not spoil the global accuracy of the scheme while ensuring stability.

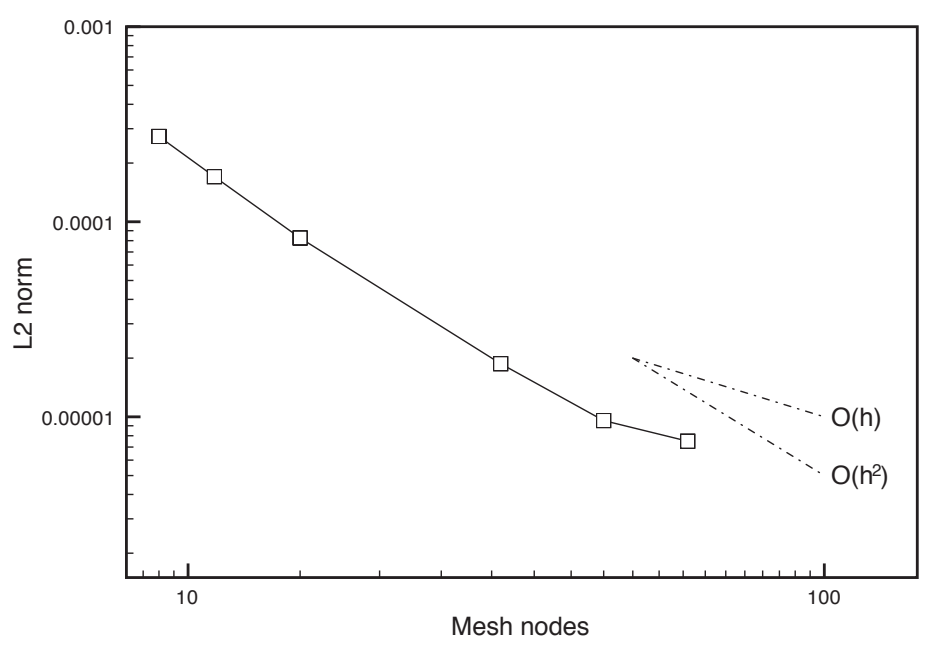

Figure 7. NSCBC method: order of convergence of $L^{2}$-norm density error (40) with respect to space discretization.

Higher-order boundary conditions A possible method to avoid problems described in the previous section would be to have second-order accuracy at the boundaries to reduce dissipation. Moreover, in the case of simulations using higher-order schemes (more than secondorder within the computational domain), the first-order numerical boundary scheme can spoil Copyright (c) 2000 John Wiley \& Sons, Ltd. Int. J. Numer. Meth. Fluids 2000; 00:1-6 Prepared using fldauth.cls 
the global accuracy of the method, which is not desirable even though robustness is ensured. Indeed, dissipation introduced by $\mathrm{ABC}$ terms is then much too high at the walls and may destroy the benefits of using a high-order scheme within the domain.

To determine the importance of wall BC versus inner domain scheme accuracy, the test case of Figure 2 was run again using the same wall BC but a more precise numerical scheme within the domain. The discretization is now a fourth-order accurate explicit centred scheme with fourthorder accurate Runge-Kutta time marching method, together with NSCBC formulation and a first-order numerical boundary scheme at the walls. Except for the numerical scheme, the test case remains the same. While the inner discretization shows great accuracy, the dissipation of the pressure signal is quite close to that obtained with TG2 scheme (Figure 6 - left). This test confirms that the dissipation of the acoustic wave observed in Figure 6 - left or 8 is not due to the inner scheme but mainly to the wall BC, highlighting the importance of wall treatment.

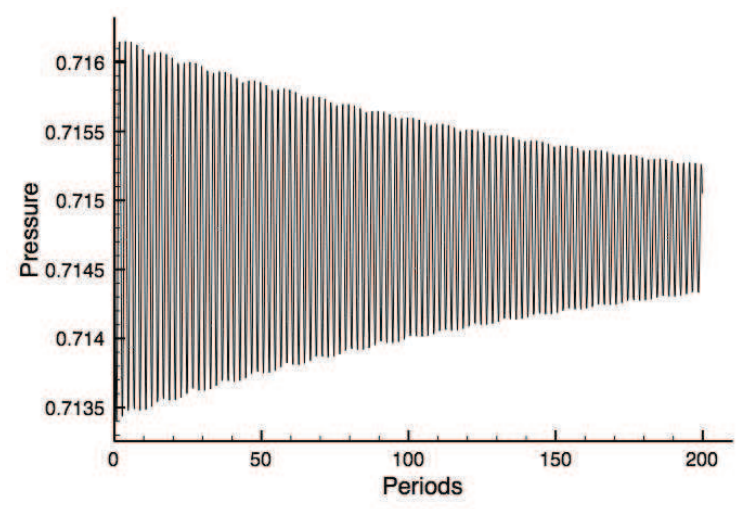

Figure 8. Pressure fluctuations at the probe of Fig. 2. Inner discretization: $4^{\text {th }}$-order explicit centred scheme and NSCBC formulation with $1^{\text {st }}$-order numerical boundary scheme. CFL number: 0.5

An obvious solution to improve the results of Figure 6 - left or 8 is then to change the numerical Copyright (C) 2000 John Wiley \& Sons, Ltd. Int. J. Numer. Meth. Fluids 2000; 00:1-6 Prepared using fldauth.cls 
boundary scheme used for first spatial derivatives. For example, approximations (23) and (24) can be respectively replaced by:

$$
\begin{aligned}
& \left.\frac{\partial \mathbf{U}}{\partial x}\right|_{0} \approx \frac{1}{2 h}\left(-\mathbf{U}_{2}+4 \mathbf{U}_{1}-3 \mathbf{U}_{0}\right) \quad \text { and }\left.\quad \frac{\partial \mathbf{U}}{\partial n}\right|_{0}=-\left.\frac{\partial \mathbf{U}}{\partial x}\right|_{0} \\
& \left.\frac{\partial \mathbf{U}}{\partial x}\right|_{N} \approx \frac{1}{2 h}\left(3 \mathbf{U}_{N}-4 \mathbf{U}_{N-1}+\mathbf{U}_{N-2}\right) \quad \text { and }\left.\quad \frac{\partial \mathbf{U}}{\partial n}\right|_{N}=+\left.\frac{\partial \mathbf{U}}{\partial x}\right|_{N}
\end{aligned}
$$

As expected, the complete scheme is then much less dissipative (eigenvalues with modulus closer to 1 - see Figure 9). Nevertheless, robustness is also shrunk: CFL number must be less than 0.1 , otherwise eigenvalues with modulus strictly greater than 1 appear in the spectrum and numerical instabilities appear in the calculation.
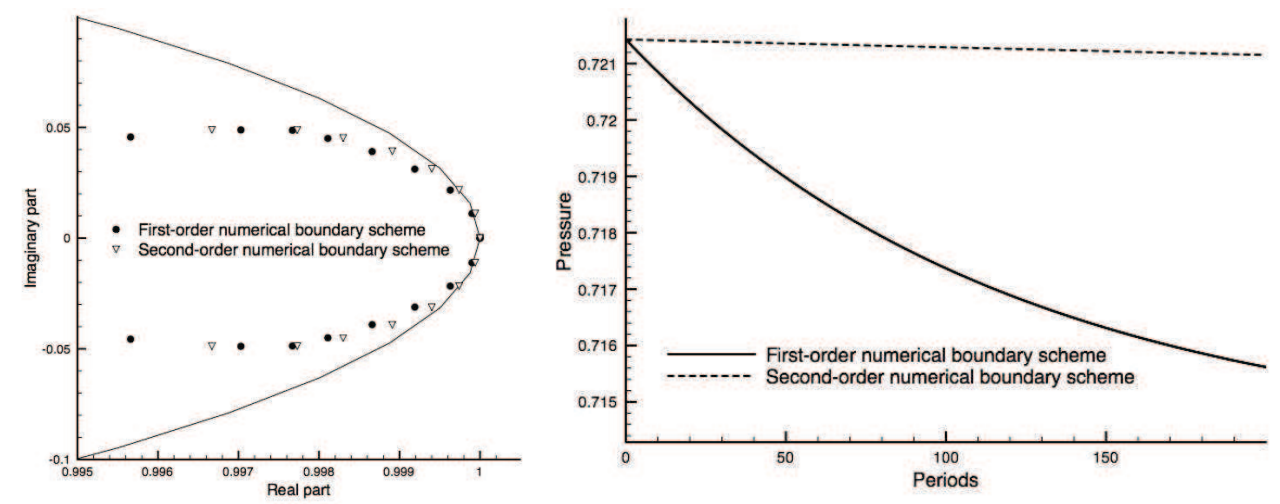

Figure 9. Effect of high-order wall BC. Left: zoom on eigenvalues with modulus equal or close to 1. Right: Predictions with $\left|\lambda_{K}\right|$. CFL number: 0.05

Indeed approximations (41) and (42) are second-order accurate, which implies that the first diffusive term of the truncation error of $\mathrm{ABC}$ terms is now a fourth derivative (which is proportional to $h^{3}$ ). They are still dissipative and stabilizing (which remains an interesting feature), though far less than approximations (38) - (39). Thus, while increasing the order of Copyright (C) 2000 John Wiley \& Sons, Ltd. Int. J. Numer. Meth. Fluids 2000; 00:1-6 Prepared using fldauth.cls 
accuracy of the numerical boundary scheme has limited interest for TG2 scheme $\|$, it should be considered with care for higher-order methods.

\section{A SIMPLE MULTIDIMENSIONAL EXAMPLE}

In this section, the evolution of a simple 2D longitudinal / transversal acoustic mode in a duct is presented as a demonstration of the stabilizing effect of the NSCBC method in a multidimensional configuration.

\subsection{Infinite duct}

As an illustration, consider a propagating longitudinal acoustic mode ( $y$-direction) with a transverse structure ( $x$-direction) in a duct, as shown on Figure 10.

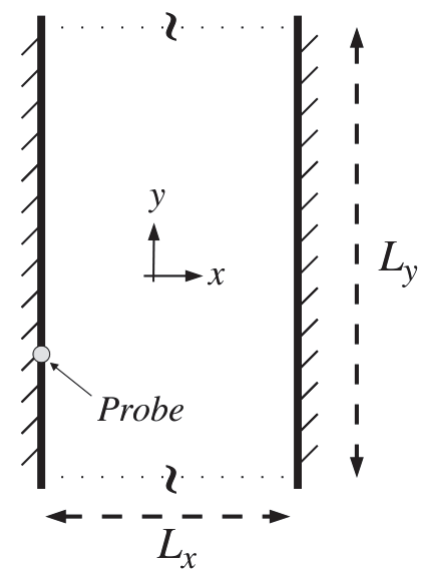

Figure 10. Sketch of the two-dimensional duct problem. $y$-direction is periodic.

\footnotetext{
\| The gain of accuracy is sensible after a long time, but the scheme is not as stable and in the case of unstructured grids, the numerical boundary scheme is complicated to express.

Copyright (C) 2000 John Wiley \& Sons, Ltd.

Int. J. Numer. Meth. Fluids 2000; 00:1-6

Prepared using fldauth.cls
} 
The solution of such an eigenmode is given by [32]:

$$
\begin{aligned}
p^{\prime}(x, y, t) & =p_{11} \cos \left(\frac{\pi x}{L_{x}}\right) \sin \left(k_{y} y-\omega t\right), \\
u^{\prime}(x, y, t) & =\frac{p_{11} \pi}{\bar{\rho} L_{x} \omega} \sin \left(\frac{\pi x}{L_{x}}\right) \cos \left(k_{y} y-\omega t\right), \\
v^{\prime}(x, y, t) & =-\frac{p_{11} k_{y}}{\bar{\rho} \omega} \cos \left(\frac{\pi x}{L_{x}}\right) \cos \left(k_{y} y-\omega t\right),
\end{aligned}
$$

with $p_{11}$ a real constant, $L_{x}$ the duct width, $k_{y}$ the wave number in direction $y$ and $\omega=2 \pi / T$ the frequency. Left and right boundaries are solid slipping walls handled with either Dirichlet or characteristic methods. The computational domain $\Omega$ is periodic in direction $y$ (infinite duct), $L_{y}=2 L_{x}$ and $k_{y}=k_{x}=\pi$. This choice of a periodic duct rather than a squared cavity bordered with solid walls is motivated by the will to avoid corners, which could bias the results and need special treatment [29]. $\Omega$ is discretized with regular $P 1$ triangles (15 nodes in $x$-direction, 28 in $y$-direction) and the numerical scheme is still TG2. CFL number is 0.5. The initial condition is given by Eqs (43)-(45) with $t=0$.

\subsection{Results}
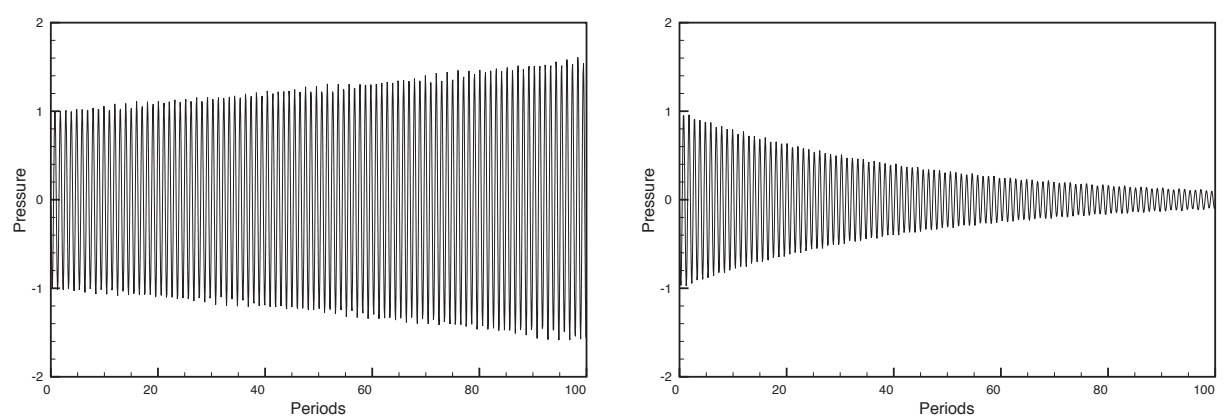

Figure 11. Pressure fluctuations at the probe of Fig. 10. Left: Dirichlet BC, right: characteristic BC. 
Figure 11 gives a comparison between the pressure signal on a wall (Figure 10) for simulations run with walls treated either with Dirichlet or characteristic BC. It is clear that the pressure fluctuation diverge when Dirichlet BC are used, while it is damped when walls are handled with characteristic BC.
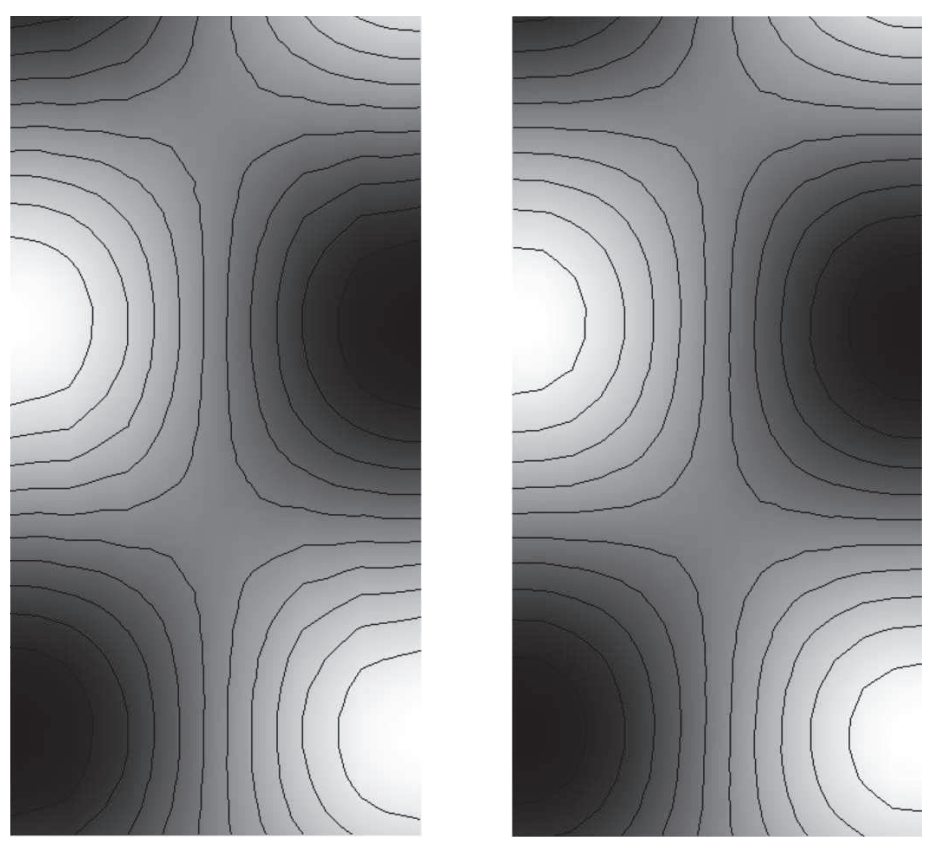

Figure 12. Pressure fluctuation magnitude for $t=8 T$. Left: Dirichlet BC (white: 0, black: 0.93), right: characteristic BC (white: 0, black: 0.88).

As shown on Figure 12, computations using either Dirichlet or characteristic wall BC give quite similar pressure fluctuations for short times. The fields are close and there is only a little difference in amplitude. Moreover, the two calculations fit the exact solution well, aside from a little small phase error. For longer times, the physical sinusoidal solution is still retrieved in the simulation with characteristic wall $\mathrm{BC}$ (there is obviously some phase error due to the numerical scheme and spurious damping mainly because of the ABC term - see Figure 13 Copyright (c) 2000 John Wiley \& Sons, Ltd.

Int. J. Numer. Meth. Fluids 2000; 00:1-6 Prepared using fldauth.cls 
right) and the computation is stable and safe from numerical instabilities. On the other hand, the simulation with Dirichlet BC eventually suffer from a non-linear behaviour and strong numerical instabilities that lead to its crash (Figure 13 - left).
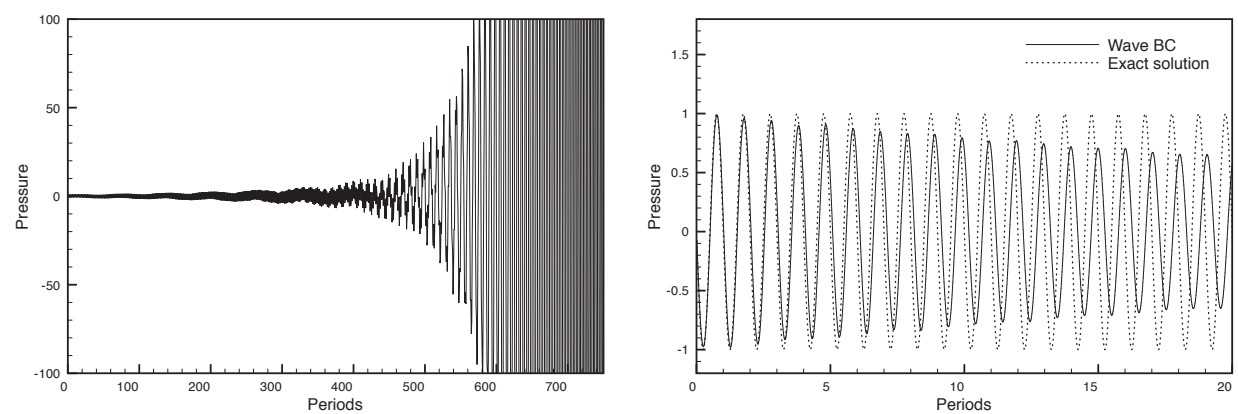

Figure 13. Pressure fluctuations at the probe of Fig. 10. Left: Dirichlet BC, on a longer span of time, right: characteristic $\mathrm{BC}$ versus exact solution (zoom on the first 20 periods).

To sum things up, the conclusions drawn in the preceding sections with one-dimensional analyses also holds in multidimensional studies: Dirichlet and characteristic BC are no more equivalent as soon as the equations are discretized. While the first can provoke strong numerical instabilities and may lead to the crash of simulations, the second have a stabilizing effect.

\section{CONCLUSION}

Building boundary conditions at walls in compressible simulation is a difficult topic if one wants to obtain both robustness and limited dissipation. This paper provides a comparison between two methods to treat solid walls in compressible flow simulations. The first is based on Dirichlet (or Neumann) formulation, which consists in imposing a zero normal velocity (or a zero pressure gradient) at the wall, while the second is based on characteristic wave Copyright (c) 2000 John Wiley \& Sons, Ltd. Int. J. Numer. Meth. Fluids 2000; 00:1-6 Prepared using fldauth.cls 
decomposition (NSCBC method). Even though they are supposed to be physically equivalent, they do not solve the same discretized equations. Theoretical analysis gives rise to additional pressure terms in the continuity equation in the case of characteristic $\mathrm{BC}$, called here $\mathrm{ABC}$ terms. After discretization, it is shown that $\mathrm{ABC}$ terms have a stabilizing effect, which is a very desirable property in non-linear simulations. Numerical calculations and amplification matrix eigenvalues confirm the increased robustness of NSCBC formulation.

While it does not seem to be a serious issue, the stabilizing effect can be too strong when dealing with higher-order methods because of the dissipation induced by ABC terms. In such a case, higher-order approximations have to be considered, which is not a problem when the mesh is structured but can be a harder task for unstructured meshes.

In terms of applications, these results show that Dirichlet methods at walls should not be used in configurations where the acoustic damping of cavities is computed. These methods will lead to wrong damping coefficients and sometimes to numerical instabilities. Using characteristic formulation such as NSCBC leads to stable schemes but also to a dissipation level which is controlled by the order of accuracy used for wall derivatives much more than by the precision of the numerical scheme within the domain. This means that wall treatments will be very important in such compressible flows.

\section{APPENDIX}

This section presents the terms of matrix $Q$ for Dirichlet and NSCBC formulations. Solution vector $\mathbf{U} \in \mathbb{R}^{2 N+2}$ and is written:

$$
\mathbf{U}=\left(\rho_{0}, u_{0}, \rho_{1}, u_{1}, \ldots, \rho_{J}, u_{J}, \ldots, \rho_{N-1}, u_{N-1}, \rho_{N}, u_{N}\right)^{T}
$$

Copyright (c) 2000 John Wiley \& Sons, Ltd.

Int. J. Numer. Meth. Fluids 2000; 00:1-6

Prepared using fldauth.cls 
Let us decompose the matrix in three parts:

$$
Q=Q_{c}+Q_{l}+Q_{r}
$$

where $Q_{c}$ is a square matrix for the discretization for degrees of freedom $\left(u_{1}, \rho_{2}, \ldots, \rho_{N-2}, u_{N-2}, u_{N-1}\right)$,

$Q_{l}$ and $Q_{r}$ are respectivelly containing the left and right numerical boundary schemes, with BC.

Dirichlet $B C$

As $u_{0}$ and $u_{N}$ are imposed to be zero, they are not degrees of freedom anymore. As a consequence, the corresponding lines in vector $\mathbf{U}$ and matrix $Q$ are suppressed, then $\mathbf{U} \in \mathbb{R}^{2 N}$ and $Q \in \mathcal{M}_{2 N}(\mathbb{R})$. We give discretization for degrees of freedom $\left(\rho_{0}, \rho_{1}, u_{1}\right)$ and $\left(\rho_{N-1}, u_{N-1}, \rho_{N}\right)$. The other lines are completed with zeros. Then, using equations (20), (21), (29) and (30) gives:

$$
Q_{l}=\left(\begin{array}{cccccccc}
1 & 0 & -\frac{\bar{\rho} \Delta t}{h} & 0 & \ldots & \ldots & \ldots & 0 \\
\frac{1}{2} \frac{c^{2} \Delta t^{2}}{h^{2}} & 1-\frac{c^{2} \Delta t^{2}}{h^{2}} & 0 & \frac{1}{2} \frac{c^{2} \Delta t^{2}}{h^{2}} & -\frac{\bar{\rho} \Delta t}{2 h} & 0 & \ldots & 0 \\
\frac{c^{2}}{\bar{\rho}} \frac{\Delta t}{2 h} & 0 & 1-\frac{c^{2} \Delta t^{2}}{h^{2}} & -\frac{c^{2}}{\bar{\rho}} \frac{\Delta t}{2 h} & \frac{1}{2} \frac{c^{2} \Delta t^{2}}{h^{2}} & 0 & \ldots & 0 \\
0 & \ldots & \ldots & \ldots & \ldots & \ldots & \ldots & 0 \\
\ldots & \ldots & \ldots & \ldots & \ldots & \ldots & \ldots & \ldots \\
0 & \ldots & \ldots & \ldots & \ldots & \ldots & \ldots & 0
\end{array}\right)
$$

and

$$
Q_{r}=\left(\begin{array}{cccccccc}
0 & \ldots & \ldots & \ldots & \ldots & \ldots & \ldots & 0 \\
. . & \ldots & \ldots & \ldots & \ldots & \ldots & \ldots & \ldots \\
0 & \ldots & \ldots & \ldots & \ldots & \ldots & \ldots & 0 \\
0 & \ldots & 0 & \frac{1}{2} \frac{c^{2} \Delta t^{2}}{h^{2}} & \frac{\bar{\rho} \Delta t}{2 h} & 1-\frac{c^{2} \Delta t^{2}}{h^{2}} & 0 & \frac{1}{2} \frac{c^{2} \Delta t^{2}}{h^{2}} \\
0 & \ldots & 0 & \frac{c^{2}}{\bar{\rho}} \frac{\Delta t}{2 h} & \frac{1}{2} \frac{c^{2} \Delta t^{2}}{h^{2}} & 0 & 1-\frac{c^{2} \Delta t^{2}}{h^{2}} & -\frac{c^{2}}{\bar{\rho}} \frac{\Delta t}{2 h} \\
0 & \ldots & \ldots & \ldots & \ldots & 0 & \frac{\bar{\rho} \Delta t}{h} & 1
\end{array}\right)
$$




\section{$N S C B C$}

The same method is used for NSCBC formulation. In this case, $u_{0}$ and $u_{N}$ are given by relations (32) and (34). Therefore, $\mathbf{U} \in \mathbb{R}^{2 N+2}$ and $Q \in \mathcal{M}_{2 N+2}(\mathbb{R})$. For this formulation, only discretizations for unknowns $\left(\rho_{0}, u_{0}\right)$ and $\left(\rho_{N}, u_{N}\right)$ are stored in $Q_{l}$ and $Q_{r}$. Using (31) and (33) yields:

$$
\begin{aligned}
& Q_{l}=\left(\begin{array}{cccccccccc}
1-\frac{c \Delta t}{h} & \frac{\bar{\rho} \Delta t}{h} & \frac{c \Delta t}{h} & -\frac{\bar{\rho} \Delta t}{h} & 0 & \ldots & \ldots & \ldots & \ldots & 0 \\
0 & 1 & 0 & 0 & 0 & \ldots & \ldots & \ldots & \ldots & 0 \\
0 & \ldots & \ldots & \ldots & \ldots & \ldots & \ldots & \ldots & \ldots & 0 \\
\ldots & \ldots & \ldots & \ldots & \ldots & \ldots & \ldots & \ldots & \ldots & \ldots \\
0 & \ldots & \ldots & \ldots & \ldots & \ldots & \ldots & \ldots & \ldots & 0
\end{array}\right) \\
& Q_{r}=\left(\begin{array}{cccccccccc}
0 & \ldots & \ldots & \ldots & \ldots & \ldots & \ldots & \ldots & \ldots & 0 \\
. . & \ldots & \ldots & \ldots & \ldots & \ldots & \ldots & \ldots & \ldots & \ldots \\
0 & \ldots & \ldots & \ldots & \ldots & \ldots & \ldots & \ldots & \ldots & 0 \\
0 & \ldots & \ldots & \ldots & \ldots & 0 & \frac{c \Delta t}{h} & \frac{\bar{\rho} \Delta t}{h} & 1-\frac{c \Delta t}{h} & -\frac{\bar{\rho} \Delta t}{h} \\
0 & \ldots & \ldots & \ldots & \ldots & 0 & 0 & 0 & 0 & 1
\end{array}\right)
\end{aligned}
$$

Matrix $Q_{c}$

Matrix $Q_{c}$ has the size of $Q_{l}$ and $Q_{r}$ and is filled using (20) and (21), except for its first and last lines** which contain only zeros (those corresponding to non-zero lines of $Q_{l}$ and $Q_{r}$ ).

** Dirichlet BC: the first and last three lines are zero; NSCBC: The first and last two lines are zero. 


$$
Q_{c}=\left(\begin{array}{cccccccccc}
0 & \ldots & \ldots & \ldots & \ldots & \ldots & \ldots & \ldots & \ldots & 0 \\
\ldots & \ldots & \ldots & \ldots & \ldots & \ldots & \ldots & \ldots & \ldots & \ldots \\
\ldots & 0 & \frac{1}{2} \frac{c^{2} \Delta t^{2}}{h^{2}} & \frac{\bar{\rho} \Delta t}{2 h} & 1-\frac{c^{2} \Delta t^{2}}{h^{2}} & 0 & \frac{1}{2} \frac{c^{2} \Delta t^{2}}{h^{2}} & -\frac{\bar{\rho} \Delta t}{2 h} & 0 & \ldots \\
\ldots & 0 & \frac{c^{2}}{\bar{\rho}} \frac{\Delta t}{2 h} & \frac{1}{2} \frac{c^{2} \Delta t^{2}}{h^{2}} & 0 & 1-\frac{c^{2} \Delta t^{2}}{h^{2}} & -\frac{c^{2}}{\bar{\rho}} \frac{\Delta t}{2 h} & \frac{1}{2} \frac{c^{2} \Delta t^{2}}{h^{2}} & 0 & \ldots \\
\ldots & \ldots & \ldots & \ldots & \ldots & \ldots & \ldots & \ldots & \ldots & \ldots \\
0 & \ldots & \ldots & \ldots & \ldots & \ldots & \ldots & \ldots & \ldots & 0
\end{array}\right)
$$

\section{ACKNOWLEDGEMENTS}

First author was financially supported by Turbomeca-Safran Group and second author's work was part of the fellow training exchange program in the framework of the Marie Curie RTN FLUISTCOM (FLUId-STructure interaction for COMbustion systems) project. The support of CINES and IDRIS is also greatly acknowledged.

\section{REFERENCES}

1. Pope SB. Turbulent flows. Cambridge University Press, 2000.

2. Selle L, Lartigue G, Poinsot T, Koch R, Schildmacher KU, Krebs W, Prade B, Kaufmann P, Veynante

D. Compressible Large-Eddy Simulation of turbulent combustion in complex geometry on unstructured meshes. Combust. Flame 2004; 137:489-505.

3. Boudier G, Gicquel LYM, Poinsot T, Bissières D, Bérat C. Comparison of LES, RANS and Experiments in an Aeronautical Gas Turbine Combustion Chamber. Proc. of the Combustion Institute 2007; 31:30753082 .

4. Bodony DJ, Lele SK. Current Status of Jet Noise Predictions Using Large-Eddy Simulation. AIAA Journal 2008; 46:364-379.

5. Boger C, Bailly C., Juvé D. Numerical Simulation of Sound Generated by Vortex Pairing in a Mixing Layer. AIAA Journal, 2000; 38:2210-2218.

6. Bogey C, Bailly C. Large eddy simulations of transitional round jets: Influence of the Reynolds number on flow development and energy dissipation. Phys. Fluids 2006; 18:1-14. 
7. Menon S, Patel N. Subgrid modeling for simulation of spray combustion in large scale combustors. $A I A A$ Journal 2006; 44:709-723.

8. Moin P, Apte SV. Large-eddy simulation of realistic gas turbine combustors. AIAA Journal 2006; 44:698708.

9. Boileau M, Staffelbach G, Cuenot B, Poinsot T, Bérat C. LES of an ignition sequence in a gas turbine engine. Combust. Flame 2008; 154:2-22.

10. Patel N, Menon S. Simulation of spray-turublence-flame interactions in a lean direct injection combustor. Combust. Flame 2008; 153:228-257.

11. Ferziger J, Perić M. Computational Methods for Fluid Dynamics. Springer Verlag, 1997.

12. Colin O, Rudgyard M. Development of high-order Taylor-Galerkin schemes for unsteady calculations. J. Comput. Phys. 2000; 162:338-371.

13. Sengupta T. Fundamentals of Computational Fluid Dynamics. Universities Press, 2004.

14. Sengissen A, Giauque A, Staffelbach G, Porta M, Krebs W, Kaufmann P, Poinsot T. Large Eddy Simulation of piloting effects on turbulent swirling flames. Proc. of the Combustion Institute 2007; 31:1729-1736.

15. Lele S. Compact finite difference schemes with spectral like resolution. J. Comput. Phys. 1992; 103:16-42.

16. Carpenter M, Gottlieb D, Abarbanel S. The Stability of Numerical Boundary Treatments for Compact High-Order Finite-Difference Schemes. J. Comput. Phys. 1993; 108:272-295.

17. Sengupta TK, Ganerwal G. Analysis of central and upwind compact schemes. J. Comput. Phys. 2003; 192:677-694.

18. Morinishi Y, Lund T, Vasilyev O, Moin P. Fully Conservative Higher Order Finite Difference Schemes for Incompressible Flow. J. Comput. Phys. 1998; 143:90-124.

19. Donea J. A Taylor-Galerkin method for convective transport problems. Int. J. for Num. Meth. in Fluids $1984 ; 20: 101-119$.

20. Thompson K. Time dependent boundary conditions for hyperbolic systems, II. J. Comput. Phys. 1990; 89:439-461.

21. Poinsot T, Lele S. Boundary conditions for direct simulations of compressible viscous flows. J. Comput. Phys. 1992; 101:104-129.

22. Carpenter M, Gottlieb D, Abarbanel S. Time stable boundary conditions for finite difference schemes solving hyperbolic systems: methodology and application to high order schemes. J. Comput. Phys. 1994; 111:220-236.

23. Nicoud F, Winckelmans G, Carati D, Baggett J, Cabot W. Boundary conditions for LES away from the

Copyright (c) 2000 John Wiley \& Sons, Ltd. Int. J. Numer. Meth. Fluids 2000; 00:1-6

Prepared using fldauth.cls 
wall. Summer Program, Center for Turbulence Research, 1998; 413-422.

24. Nicoud F. Defining wave amplitude in characteristic boundary conditions. J. Comput. Phys. 1998; 149:418-422.

25. Yoo CS, Im HG. Characteristic Boundary Conditions for Simulations of Compressible Reacting Flows with Multi-Dimensional,Viscous, and Reaction Effects. Combust. Theory and Modelling 2007; 11:259-286.

26. Selle L, Nicoud F, Poinsot T. The actual impedance of non-reflecting boundary conditions: implications for the computation of resonators. AIAA Journal 2004; 42:958-964.

27. Prosser, R Improved Boundary conditions for the direct numerical simulation of turbulent subsonic flows I: Inviscid flows. J. Comput. Phys. 2005; 207:736-768.

28. Prosser, R Towards improved boundary conditions for the DNS and LES of turbulent subsonic flows. J. Comput. Phys. 2005; 222:469-474.

29. Lodato G, Domingo P, Vervisch L. Three-dimensional boundary condtions for direct and large-eddy simulation of compressible viscous flow. J. Comput. Phys. 2008; .

30. Schmitt P, Poinsot T, Schuermans B, Geigle K. Large-eddy simulation and experimental study of heat transfer, nitric oxide emissions and combustion instability in a swirled turbulent high pressure burner. J. Fluid Mech. 2007; 570:17-46.

31. Wagner C, Hüttl T, Sagaut P ( (eds.)). Large-Eddy Simulation for Acoustics. Cambridge University Press, 2007.

32. Poinsot T, Veynante D. Theoretical and numerical combustion. R.T. Edwards, 2nd edition., 2005.

33. Moureau V, Lartigue G, Sommerer Y, Angelberger C, Colin O, Poinsot T. Numerical methods for unsteady compressible multi-component reacting flows on fixed and moving grids. J. Comput. Phys. 2004; 202:710736.

34. Thompson KW. Time dependent boundary conditions for hyperbolic systems. J. Comput. Phys. 1987; 68:1-24.

35. Colonius, T. and Lele, S. and Moin, P. Boundary conditions for direct computation of aerodynamic sound generation. AIAA Journal 1993; 31:1574-1582

36. Guichard L, Réveillon J, Hauguel R. Direct numerical simulation of statistically stationary one and twophase turbulent combustion : a turbulent injection procedure. Flow, Turbulence and Combustion 2004; 73:133-167.

37. Baum M, Poinsot TJ, Thévenin D. Accurate boundary conditions for multicomponent reactive flows. J. Comput. Phys. 1994; 116:247-261.

Copyright (C) 2000 John Wiley \& Sons, Ltd. Int. J. Numer. Meth. Fluids 2000; 00:1-6

Prepared using fldauth.cls 
38. Löhner R, Morgan K, Zienkiewicz O. An adaptative finite element procedure for high speed flows. Comp. Meth. Appl. Mech. E Eng. 1985; 51:441-465.

39. Donea J, Huerta A. Finite Element Methods for Flow Problems. Wiley, 2003.

40. Lax PD, Wendroff B. Systems of conservation laws. Comm. Pure Appl. Math. 1960; 13:217-237.

41. Hirsch C. Numerical Computation of internal and external flows, vol. 1. John Wiley: New York, 1988.

42. Warming R, Beam R. An Eigenvalue Analysis of Finite-Difference Approximations for Hyperbolic IBVPs. Technical Report TM 102241, NASA 1989.

43. B Gustafsson. The convergence rate for difference approximations to mixed initial boundary value problems. Math. Comput. 1975; 29:396-406. 